\title{
An Application of the Stiefel-Whitney Classes to the Proof of a Fixed Point Theorem for Set-Valued Mappings
}

by

\author{
Dariusz MIKLASZEWSKI
}

Presented by Andrzej LASOTA

Summary. We prove a fixed point theorem for Borsuk continuous mappings with spherical values, which extends a previous result. We apply some nonstandard properties of the Stiefel-Whitney classes.

1. Introduction. The aim of this note is to complete the proof of the following result for set-valued mappings of the disc $D^{n}$ in the $n$-dimensional Euclidean space.

THEOREM 1. Let $f: D^{n} \rightarrow 2^{D^{n}}$ be a Borsuk continuous map such that $n \neq 6$ and for every $x$ in $D^{n}, f(x)$ is homeomorphic to either a point or the $(n-2)$-sphere $S^{n-2}$. Then $f$ has a fixed point.

Borsuk continuity means continuity with respect to the metric $\varrho_{c}$ defined in [1]. Our research is motivated by Górniewicz's fixed point theorem for Borsuk continuous mappings of $D^{2}$ (see [6]) and Schirmer's results on bimaps with values homeomorphic to a point or $S^{0}$ (see [16]). The author does not know if $S^{n-2}$ in Theorem 1 can be replaced by $S^{k}$ with $1 \leq k \leq n-3$.

Theorem 1 was proved in [12] for $n=3$ and in [13] for $n=3,4,5$. For completeness we first recall the sketch of the proof of Theorem 1 in a special case. In this case all bundles which appear in the proof are assumed to have the structural group $O(n-1)$. We point out those steps of the proof which seem to be difficult without this restriction. Then we show how to avoid the difficulties.

2000 Mathematics Subject Classification: 55M20, 55R25, 54C60.

Key words and phrases: fixed points of set-valued mappings, Stiefel-Whitney classes, Borsuk continuity. 
2. Sketch of the proof of Theorem 1 in a special case. Let $U=$ $\left\{x \in D^{n}: f(x) \cong S^{n-2}\right\}$. The set $U$ is open in $D^{n}$. Roughly speaking, we approximate $\partial U$ by some $(n-1)$-dimensional closed connected manifolds $M$ in $U$ (see [11, pp. 56-57]). Fix such an $M$. Let $\Gamma=\Gamma_{M}$ denote the graph of $\left.f\right|_{M}$. We need the assumption $n \neq 6$ to deduce from the Borsuk continuity of $f$ that the projection $p: \Gamma \rightarrow M$ is a completely regular map and locally trivial fibration [4]. Here we apply Ferry's theorem on approximating homotopy equivalences by homeomorphisms, which seems to be unknown in dimension 4 (see [2], [5], [8], [9]).

Denote the Stiefel-Whitney classes of the bundle $p$ with fibre $S^{n-2}$ by $w_{1}, \ldots, w_{n-1}$. The Stiefel-Whitney classes are defined in terms of the Thom isomorphism for all spherical fibrations (see for instance [10, p. 181]). By [11, Theorem 1], a sufficient condition for $f$ to have a fixed point is that

$$
\operatorname{dim} H_{n-2}\left(\Gamma ; Z_{2}\right)>\operatorname{dim} H_{n-2}\left(M ; Z_{2}\right),
$$

which is equivalent to the equality

$$
w_{n-1}=0 \quad(\text { see }[12, \text { p. 128]). }
$$

- The first restriction for this special case consists in assuming that there is a free fibre-preserving action of $Z_{2}$ on $\Gamma$ such that the orbit spaces of the fibres are the projective spaces $\mathbb{R} P^{n-2}$. If we try to define such an action in the natural way, i.e. transporting the antipodal map of $S^{n-2}$ to the fibres by local trivializations, then we succeed only if all mappings in the structural group of $p$ are odd.

Having the above action we proceed as follows. Let $c$ be the first (= the last) Stiefel-Whitney class of the bundle $\Gamma \rightarrow \Gamma / Z_{2}$. Let $q: \Gamma / Z_{2} \rightarrow M$ be the map induced by $p$. Then

$$
c^{n-1}=\sum_{j=1}^{n-1} q^{\star}\left(\widetilde{w}_{j}\right) \cup c^{n-1-j} \quad \text { for some } \widetilde{w}_{1}, \ldots, \widetilde{w}_{n-1},
$$

by the Leray-Hirsch theorem (see [7, III.1]); and

$$
c^{n}=0,
$$

since otherwise the projection $\Gamma \subset M \times \mathbb{R}^{n} \rightarrow \mathbb{R}^{n}$ contradicts a version of the Borsuk-Ulam theorem (see [13, pp. 73-74]).

- The second condition, which should be clarified in the general case, is the equality $\widetilde{w}_{j}=w_{j}$ for $j=1, \ldots, n-1$. Proofs of this equality, as far as I know, use the Splitting Principle, which is a property of the bundles having the structural group $O(n-1)$ [7, III.5]. We assume that this is the case. 
From (1), (2) and the above assumption we have

$$
0=c^{n-1} \cup c=\sum_{j=1}^{n-2} q^{\star}\left(w_{1} \cup w_{j}+w_{j+1}\right) \cup c^{n-j-1} .
$$

This gives $w_{j+1}=w_{1} \cup w_{j}$ for $j=1, \ldots, n-2$, and $w_{n-1}=\left(w_{1}\right)^{n-1}=0$, the last equality being a consequence of the triviality of the $(n-1)$ th power in the $Z_{2}$-cohomology algebra of any closed $(n-1)$-dimensional manifold in $\mathbb{R}^{n}$ (see [13, pp. 72-73]). This proves the theorem (in the special case).

3. The general case. Let $S=S^{n-2}, S^{\triangle}=S \times S \backslash \triangle$ with $\triangle$ the diagonal. It is easy to check that the maps $\phi, \psi: S^{\triangle} \rightarrow S$ defined by

$$
\phi(x, y)=\|x-y\|^{-1} \cdot(x-y), \quad \psi(x, y)=x
$$

are homotopy equivalences with homotopy inverse $\omega(x)=(x,-x)$. Let

$$
\Gamma^{\triangle}=\{(x, y) \in \Gamma \times \Gamma \backslash \triangle: p(x)=p(y)\}
$$

and $p^{\triangle}(x, y)=p(x)$. The group $Z_{2}$ acts on $S^{\triangle}$ and $\Gamma^{\triangle}$ by transposition. The map $p^{\triangle}: \Gamma^{\triangle} \rightarrow M$ is a locally trivial bundle with fibre $S^{\triangle}$. The orbit spaces of the fibres of $p^{\triangle}$ are homeomorphic to $S^{\triangle} / Z_{2} \simeq \mathbb{R} P^{n-2}$, the last equivalence being induced by $\phi$ (the idea of such an equivalence is due to Cohen [3, Proposition III]). Thom proved that the Stiefel-Whitney classes are invariants of fibre homotopy equivalences [17]. Thom's argument shows that the same is true for mappings over $M$ which are homotopy equivalences on the fibres. One such mapping is

$$
\Gamma^{\triangle} \ni(x, y) \mapsto x \in \Gamma,
$$

because $\psi$ is a homotopy equivalence. Consequently, both bundles $p, p^{\triangle}$ have the same Stiefel-Whitney classes. It is a simple matter to obtain the formulae (1) and (2) from Section 2 in the new situation, with $c, q, \widetilde{w}_{j}$ replaced by $c^{\triangle} \in H^{1}\left(\Gamma^{\triangle} / Z_{2} ; Z_{2}\right), q^{\triangle}: \Gamma^{\triangle} / Z_{2} \rightarrow M$ and $w_{j}^{\triangle} \in H^{j}\left(M ; Z_{2}\right)$.

The author does not know if $w_{j}^{\triangle}=w_{j}$ for every $j$ (in spite of the fact that the mod-2 characteristic classes for spherical fibrations are indicated in [14]). Clearly, the proof of Theorem 1 can be completed along the lines of Section 2 provided

$$
w_{n-1}^{\triangle}=w_{n-1} .
$$

Our proof of this equality will resemble a classical inductive reasoning in the theory of characteristic classes, which makes use of the Gysin sequence (see $[15,14.5])$. The difference is that the splittings will have to be purely algebraic, because of the lack of the notion of orthogonal complement in the fibres. 
4. Proof of (3). To simplify notation, we continue to write

- $E=\Gamma^{\triangle}, E_{2}=\Gamma^{\triangle} / Z_{2}$;

- $w=w_{n-1}, v_{j}=w_{j}^{\triangle}, e=c^{\triangle}$;

- $r=p^{\triangle}, g=q^{\triangle}, \varrho: E \rightarrow E_{2}$ (the projection).

By the Leray-Hirsch theorem,

(*) $\quad H^{\star}\left(E_{2} ; Z_{2}\right)$ is an $H^{\star}\left(M ; Z_{2}\right)$-module freely generated by $1, e, \ldots, e^{n-2}$ with the multiplication

$$
H^{\star} M \times H^{\star} E_{2} \ni(\mu, \eta) \mapsto \mu \cdot \eta=g^{\star}(\mu) \cup \eta .
$$

The Gysin exact sequences of the bundles $r$ and $\varrho$ form the following commutative diagram:

$$
\begin{aligned}
\cdots \rightarrow H^{-1} M & \rightarrow H^{n-2} M \stackrel{r^{\star}}{\rightarrow} H^{n-2} E \stackrel{\alpha}{\rightarrow} H^{0} M \stackrel{\cup w}{\rightarrow} H^{n-1} M \stackrel{r^{\star}}{\rightarrow} H^{n-1} E \rightarrow \cdots \\
\cdots \rightarrow H^{n-3} E_{2} & \stackrel{\cup e}{\longrightarrow} H^{n-2} E_{2} \stackrel{\varrho^{\star}}{\rightarrow} H^{n-2} E \stackrel{\beta}{\longrightarrow} H^{n-2} E_{2} \stackrel{\cup e}{\longrightarrow} H^{n-1} E_{2} \stackrel{\varrho^{\star}}{\rightarrow} H^{n-1} E \rightarrow \cdots
\end{aligned}
$$

Since $H^{-1} M=0, r^{\star n-2}: H^{n-2} M \rightarrow H^{n-2} E$ is a monomorphism. Similarly, $r^{\star j}$ is an isomorphism for $j<n-2$. By our assumption, $M$ is pathwise connected, so $H^{0} M=Z_{2}$.

CASE 1. Assume that $\alpha=0$. Since $r^{\star j}$ is now an isomorphism for $j \leq$ $n-2$ and $r^{\star}=\varrho^{\star} \circ g^{\star}$, we have

$$
H^{j} E_{2}=\operatorname{ker}\left(\varrho^{\star}\right) \oplus \operatorname{im}\left(g^{\star}\right)=\operatorname{im}(\cup e) \oplus \operatorname{im}\left(g^{\star}\right)
$$

for $j \leq n-2$. Since $w=1 \cup w \in \operatorname{ker}\left(r^{\star}\right)=\operatorname{ker}\left(\varrho^{\star} \circ g^{\star}\right)$, it follows that $g^{\star} w \in \operatorname{ker}\left(\varrho^{\star}\right)=\operatorname{im}(\cup e)$. There is $x_{1} \in H^{n-2} E_{2}$ with $g^{\star} w=x_{1} \cup e$. By (4), there are $x_{2} \in H^{n-3} E_{2}$ and $v_{n-2} \in H^{n-2} M$ with $x_{1}=x_{2} \cup e+g^{\star} v_{n-2}$. By induction on $j \leq n-2$,

$$
x_{j}=x_{j+1} \cup e+g^{\star} v_{n-j-1}
$$

for some $x_{j+1} \in H^{n-2-j} E_{2}$ and $v_{n-j-1} \in H^{n-j-1} M$. Thus $g^{\star} w=x_{n-1} \cup$ $e^{n-1}+\sum_{j=1}^{n-2} g^{\star} v_{n-j-1} \cup e^{j}$ for an $x_{n-1} \in H^{0} E_{2}=\{0,1\}$. If $x_{n-1}=0$ then $(*)$ shows that $w=0$. In this way $\alpha$ is an epimorphism, $\alpha \neq 0$, which contradicts our assumption. We conclude that $x_{n-1}=1$ and

$$
e^{n-1}=g^{\star} w+\sum_{j=1}^{n-2} g^{\star} v_{n-j-1} \cup e^{j},
$$

which gives $w=v_{n-1}$ as required.

CASE 2. Assume that $\alpha \neq 0$. Thus $\alpha$ is an epimorphism, $\operatorname{ker}(\cup w)=$ $H^{0} M, w=1 \cup w=0$. 
CASE 2.1. Suppose that $\beta=0$. Hence $\varrho^{\star n-2}$ is an epimorphism. By $(*)$,

$$
H^{n-2} E_{2}=g^{\star} H^{n-2} M \oplus \bigoplus_{j=1}^{n-2}\left(g^{\star} H^{n-j-2} M\right) \cup e^{j} .
$$

Since $\varrho^{\star} \circ(\cup e)=0$, we have $\varrho^{\star} H^{n-2} E_{2}=\varrho^{\star} g^{\star} H^{n-2} M$. Thus $H^{n-2} E=$ $r^{\star} H^{n-2} M=\operatorname{ker}(\alpha), \alpha=0$, a contradiction.

CASE 2.2. Suppose that $\beta \neq 0$. There is an $x_{1} \in \operatorname{im}(\beta), x_{1} \neq 0$. Clearly, $0=x_{1} \cup e$.

CASE 2.2.1. Assume that $\alpha \varrho^{\star} x_{1}=1$. Fix $x \in H^{n-2} E$. If $\alpha(x)=0$ then $x \in \operatorname{im}\left(r^{\star}\right) \subset \operatorname{im}\left(\varrho^{\star}\right)$. If $\alpha(x)=1$ then $x=\left(x-\varrho^{\star} x_{1}\right)+\varrho^{\star} x_{1} \in \operatorname{im}\left(\varrho^{\star}\right)$, because $\alpha\left(x-\varrho^{\star} x_{1}\right)=0$. Hence $\operatorname{im}\left(\varrho^{\star}\right)=H^{n-2} E$ and $\beta=0$, a contradiction.

CASE 2.2.2. Assume that $\alpha \varrho^{\star} x_{1}=0$. Thus $\varrho^{\star} x_{1} \in \operatorname{ker}(\alpha)=\operatorname{im}\left(r^{\star}\right)=$ $\operatorname{im}\left(\varrho^{\star} \circ g^{\star}\right)$. There is a $v_{n-2} \in H^{n-2} M$ with $\varrho^{\star} x_{1}=\varrho^{\star} g^{\star} v_{n-2}$. Thus

$$
x_{1}-g^{\star} v_{n-2} \in \operatorname{ker}\left(\varrho^{\star}\right)=\operatorname{im}(\cup e) .
$$

There is an $x_{2} \in H^{n-3} E_{2}$ with $x_{1}=x_{2} \cup e+q^{\star} v_{n-2}$. We will now proceed by induction. Just as in Case $1, x_{j}=x_{j+1} \cup e+g^{\star} v_{n-j-1}$ and

$$
g^{\star} w=0=x_{1} \cup e=x_{n-1} \cup e^{n-1}+\sum_{j=1}^{n-2} g^{\star} v_{n-j-1} \cup e^{j} .
$$

Next we claim that $x_{j} \neq 0$ for every $j=1, \ldots, n-1$. Conversely, suppose that $j+1=\min \left\{m: x_{m}=0\right\}$. Thus $0=x_{1} \cup e=\sum_{i=1}^{j} g^{\star} v_{n-i-1} \cup e^{i}$. By $(*), v_{n-i-1}=0$ for $i=1, \ldots, j$. Thus $x_{j}=0$, contrary to the choice of $j$. We have proved that $x_{n-1} \neq 0$, and so $x_{n-1}=1$. Thus $e^{n-1}=g^{\star} w+$ $\sum_{j=1}^{n-2} g^{\star} v_{n-j-1} \cup e^{j}$, and $w=v_{n-1}$ as required.

The equality (3) follows and the proof of Theorem 1 is complete.

\section{References}

[1] K. Borsuk, On some metrization of the hyperspace of compact sets, Fund. Math. 41 (1954), 168-202.

[2] T. A. Chapman and S. Ferry, Approximating homotopy equivalences by homeomorphisms, Amer. J. Math. 101 (1979), 583-607.

[3] F. Cohen, Cohomology of braid spaces, Bull. Amer. Math. Soc. 79 (1973), 763-766.

[4] E. Dyer and M. E. Hamstrom, Completely regular mappings, Fund. Math. 45 (1957), 103-118.

[5] S. Ferry, Homotoping e-maps to homeomorphisms, Amer. J. Math. 101 (1979), 567582.

[6] L. Górniewicz, Present state of the Brouwer fixed point theorem for multivalued mappings, Ann. Sci. Math. Québec 22 (1998), 169-179.

[7] D. Husemoller, Fibre Bundles, McGraw-Hill, 1966. 
[8] W. Jakobsche, Approximating homotopy equivalences of surfaces by homeomorphisms, Fund. Math. 118 (1983), 1-9.

[9] -, Approximating homotopy equivalences of 3-manifolds by homeomorphisms, ibid. 130 (1988), 157-168.

[10] J. P. May, Homology operations on infinite loop spaces, in: Algebraic Topology, Proc. Sympos. Pure Math. 12, Amer. Math. Soc., 1971, 171-186.

[11] D. Miklaszewski, On the Brouwer fixed point theorem, Topology Appl. 119 (2002), $53-64$.

[12] - A fixed point theorem for multivalued mappings with nonacyclic values, Topol. Methods Nonlinear Anal. 17 (2001), 125-131.

[13] - A fixed point conjecture for Borsuk continuous set-valued mappings, Fund. Math. 175 (2002), 69-78.

[14] R. J. Milgram, The mod-2 spherical characteristic classes, Ann. of Math. 92 (1970), 238-261.

[15] J. W. Milnor and J. D. Stasheff, Characteristic Classes, Princeton Univ. Press, 1974.

[16] H. Schirmer, A fixed point index for bimaps, Fund. Math. 134 (1990), 91-102.

[17] R. Thom, Espaces fibrés en sphères et carrés de Steenrod, Ann. Sci. École Norm. Sup. 69 (1952), 109-181.

Dariusz Miklaszewski

Faculty of Mathematics and Computer Science

Nicholaus Copernicus University

Chopina 12/18

87-100 Torun, Poland

E-mail: miklasze@mat.uni.torun.pl

Received February 2, 2005;

received in final form June 11, 2005 